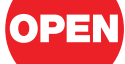

SUBJECT AREAS: BIOPHYSICS

BIOLOGICAL MODELS

LIPIDS

BIOPHYSICAL CHEMISTRY

Received

29 March 2011

Accepted

21 October 2011

Published

24 November 2011

Correspondence and requests for materials should be addressed to M.H. (hadorn@ifk.sdu.

$\mathrm{dk})$

\section{A Quantitative Analytical Method to Test for Salt Effects on Giant Unilamellar Vesicles}

\author{
Maik Hadorn', Eva Boenzli² \& Peter Eggenberger Hotz
} ${ }^{1}$ Center for Fundamental Living Technology (FLinT), Department of Physics, Chemistry and Pharmacy, University of Southern
Denmark, Odense, Denmark, ${ }^{2}$ Clinical Laboratory, Faculty of Veterinary Medicine, University of Zurich, Zurich, Switzerland, ${ }^{3}$ The
Mærsk Mc-Kinney Møller Institute, University of Southern Denmark, Odense, Denmark.

Today, free-standing membranes, i.e. liposomes and vesicles, are used in a multitude of applications, e.g. as drug delivery devices and artificial cell models. Because current laboratory techniques do not allow handling of large sample sizes, systematic and quantitative studies on the impact of different effectors, e.g. electrolytes, are limited. In this work, we evaluated the Hofmeister effects of ten alkali metal halides on giant unilamellar vesicles made of palmitoyloleoylphosphatidylcholine for a large sample size by combining the highly parallel water-in-oil emulsion transfer vesicle preparation method with automatic haemocytometry. We found that this new quantitative screening method is highly reliable and consistent with previously reported results. Thus, this method may provide a significant methodological advance in analysis of effects on free-standing model membranes.

n 1888 and 1890, Hofmeister described the ability of neutral salts to precipitate a given protein by demonstrating that distinct salts have distinct effectiveness in stabilizing protein suspensions ${ }^{1,2}$. Specific salt effects have since been attributed to a broad range of phenomena of physicochemical, biological and environment significance ${ }^{3}$.

According to the so-called Hofmeister, lyotropic or chaotropic series, anions are usually ordered as follows: $\mathrm{SO}_{4}{ }^{2-}, \mathrm{HPO}_{4}{ }^{2-}, \mathrm{OH}^{-}, \mathrm{F}^{-}, \mathrm{HCOO}^{-}, \mathrm{CH}_{3} \mathrm{COO}^{-}, \mathrm{Cl}^{-}, \mathrm{Br}^{-}, \mathrm{NO}_{3}{ }^{-}, \mathrm{I}^{-}, \mathrm{SCN}^{-}$and $\mathrm{ClO}_{4}^{-}$. There exists a broad consensus concerning anions by far dominating cations in terms of Hofmeister effects ${ }^{4}$. Nevertheless, a Hofmeister series was also reported for cations ${ }^{4-8}: \mathrm{Cs}^{+}, \mathrm{NH}_{4}{ }^{+}, \mathrm{Rb}^{+}, \mathrm{K}^{+}, \mathrm{Na}^{+}, \mathrm{Li}^{+}, \mathrm{Mg}^{2+}, \mathrm{Ca}^{2+}$ and $\mathrm{Ba}^{2+}$. Anions and cations on the left side of the two series were found to increase the solvent surface tension and decrease the solubility of nonpolar molecules. These ions are known as kosmotropes or salting-out ions. In contrast, anions and cations on the right hand side increase the solubility of nonpolar molecules, weaken the hydrophobic effect and are known as chaotropes or salting-in ions.

Despite its ubiquity, a molecular-level understanding of the Hofmeister series is still lacking. Ninham and Nostro recently summarised the current level of understanding of Hofmeister effects ${ }^{9}$. Originally, it was believed that ions affect the physical behaviour of aqueous macromolecular systems by making or breaking the water structure 4 . However, recent studies cast serious doubts on this notion. Neither was the hydrogen-bonding network significantly changed by the presence of ions, nor were ions found to have a long-range effect on bulk water structure. Evidences from work on protein/water and lipid/water interfaces suggest that bulk water structure making and breaking are not responsible for phenomena related to the Hofmeister series (for a recent review see Zhang and $\mathrm{Cremer}^{3}$ ).

Biophysical studies of salt effects on pure model bilayer systems (e.g. solid-supported membranes, freestanding artificial vesicles) suggest that salts can fundamentally alter the structure of phospholipid head groups $^{10-17}$ and that both weakly hydrated chaotropic anions and strongly hydrated, kosmotropic cations are attracted to the model membrane ${ }^{18-20}$. In general, all observed effects are ion-specific and a function of ion size, valency and polarizability ${ }^{21}$ (for a recent review, see Kunz et al. ${ }^{22}$ ). Many studies concentrated on cations, mainly focusing on the biological function and importance of biologically relevant $\mathrm{Na}^{+}, \mathrm{K}^{+}, \mathrm{Ca}^{2+}$, and $\mathrm{Mg}^{2+16,23-32}$. Anion effects on the structural properties of lipid bilayers have been examined in a rather limited and nonsystematic way ${ }^{17,33-51}$. Current analytical techniques addressing anion and cation effects on model membranes are demanding in technical requirements and are serial in design. Thus, despite the best efforts only a small fraction of effectors are testable. 
The aim of the present paper is to reduce restrictions of current protocols. To exemplify the potential of the new quantitative analytical method, we systematically investigated a wide range of alkali metal halides and quantified their effect on the preparation of zwitterionic palmitoyloleoylphosphatidylcholine (POPC) free-standing giant unilamellar vesicles (GUV). The method involved the application of the recently described water-in-oil (W/O) emulsion transfer method shown to result in unilamellar vesicles ${ }^{52}$, originally designed to prepare asymmetric vesicles ${ }^{53}$ and modified by the authors both to provide a parallel and resource-saving vesicle preparation procedure $^{54}$ and to prepare multicompartmental vesicles ${ }^{55}$, as well as of haemocytometry measurements to determine the vesicle yield and to test the reliability of the new semi-automatic quantitative analytical method described herein. In contrast to widely used flow cytometry analyses ${ }^{56,57}$, we used the impedance method for the quantification of vesicles. The effect of four alkali metal chlorides, i.e. $\mathrm{LiCl}, \mathrm{NaCl}, \mathrm{KCl}$ and $\mathrm{CsCl}$, four sodium halides, i.e. $\mathrm{NaF}, \mathrm{NaCl}$, $\mathrm{NaBr}$ and $\mathrm{NaI}$, and four potassium halides, i.e. $\mathrm{KF}, \mathrm{KCl}, \mathrm{KBr}$ and $\mathrm{KI}$, on the preparation of free-standing GUVs was tested over a concentration range of 0 to $500 \mathrm{mM}$ in seven replicas. Our results are in perfect agreement with literature. We hence conclude that the new semi-automatic quantitative analytical method is highly reliable and therefore may provide significant methodological advance in analysis of free-standing model membranes.

\section{Results}

In the negative control, vesicles were absent both using the automatic and manual vesicle counting. No significant differences, i.e. $p>0.05$, were found in the vesicle yield when comparing automatic and manual vesicle counting (Fig. 1).
The effect of halogens and alkali metals on vesicle yield was quantified by performing automatic vesicle counting with a common alkali metal cation $\left(\mathrm{Na}^{+}, \mathrm{K}^{+}\right)$or halogen anion $\left(\mathrm{Cl}^{-}\right)$. Figs. 2, 3 and 4 show the vesicle concentration in dependence of the alkali metal halide species and the ionic strength of the equiosmolar intravesicular and intervesicular solution. The matrixes below indicate significant differences using Tukey's HSD or Dunnett's T3 post hoc test.

Concerning the effect of alkali metal halides on the vesicle concentration, we observed that (i) the vesicle concentration correlates positively with the Hofmeister series for anions, i.e. down the halogen group and (ii) positively with the reverse Hofmeister series for cations, i.e. down the alkali metal group. (iii) Trends in the vesicle concentration are more prominent for anions (iv) and at a concentration of $100 \mathrm{mM}$. (v) Only iodide (i.e. NaI and KI) does not negatively affect vesicle preparation if compared to the positive control. By using light microscopy, the vesicles were found to be equal in size for all conditions tested and little to no vesicle aggregates were observed (Fig. 5).

\section{Discussion}

Because neither variations in vesicle size nor vesicle aggregates were found, the automatic and manual vesicle counting were not biased by vesicles that were not individually distinguishable, i.e. by aggregates of vesicles, or differences in vesicle size distributions. The numbers obtained therefore represent the number of individual vesicles for each condition tested.

In preliminary studies, vesicles were found to be stable both in presence and absence of electrolytes on glass surfaces treated with repel silane and in microplates. In the presence of electrolytes, vesicles undergo lysis on untreated glass surfaces; but in the absence of

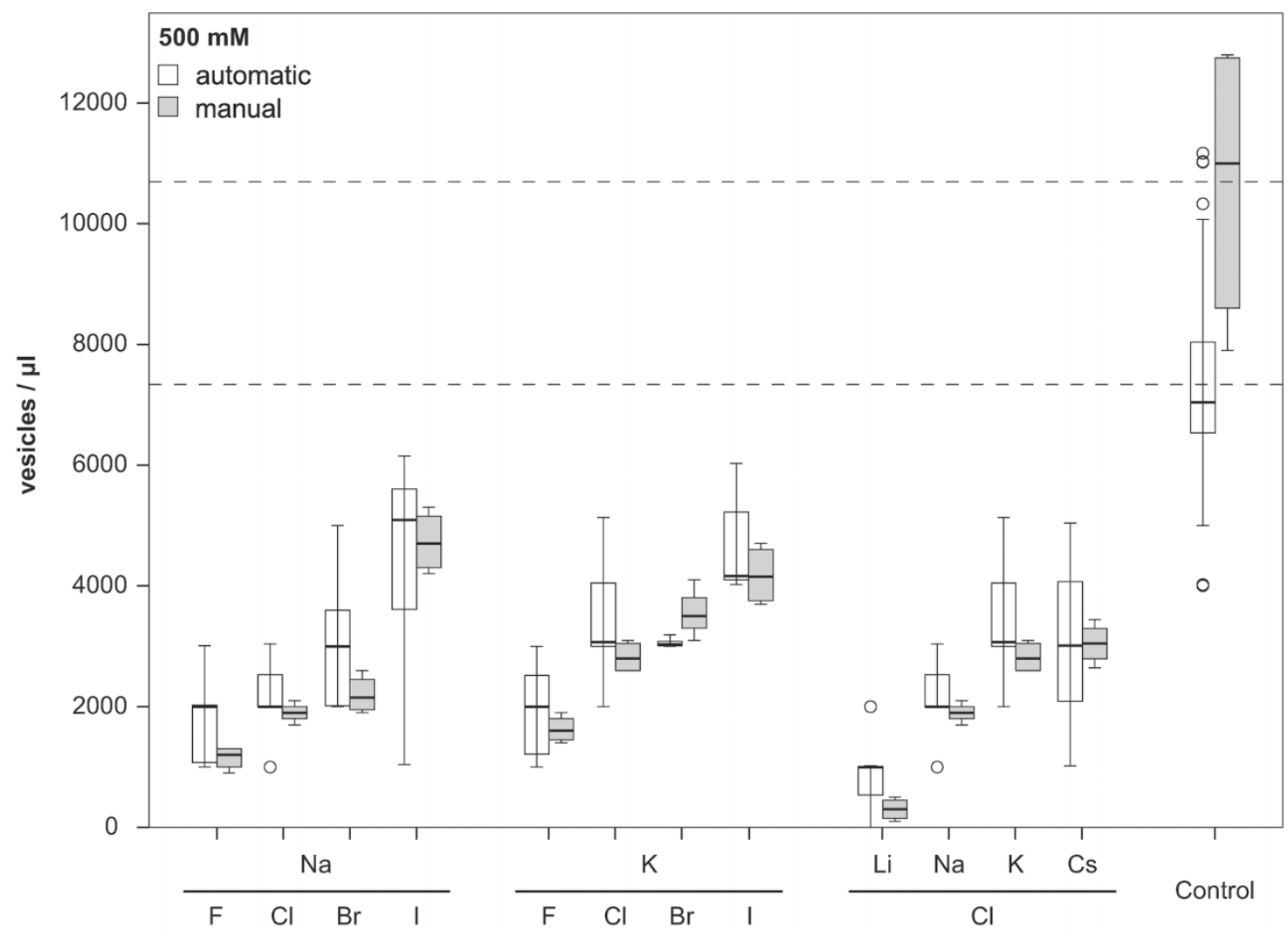

Figure 1 Comparability of automatic haemocytometric and manual counting of vesicles prepared in the presence of alkali metal halides at a concentration of $500 \mathrm{mM}$. An unpaired 2-tailed Welch's t test did not reveal any significant differences at the $p<0.05$ level. Sample sizes see text. The dashed lines represent the mean values of the positive control group, i.e. in the absence of electrolytes, of the automatic vesicle counting $\mathrm{m}_{\text {automatic }}=7337$ vesicles $/ \mu \mathrm{L}$ and the manual vesicle counting $\mathrm{m}_{\text {manual }}=10675$ vesicles $/ \mu \mathrm{L}$. 


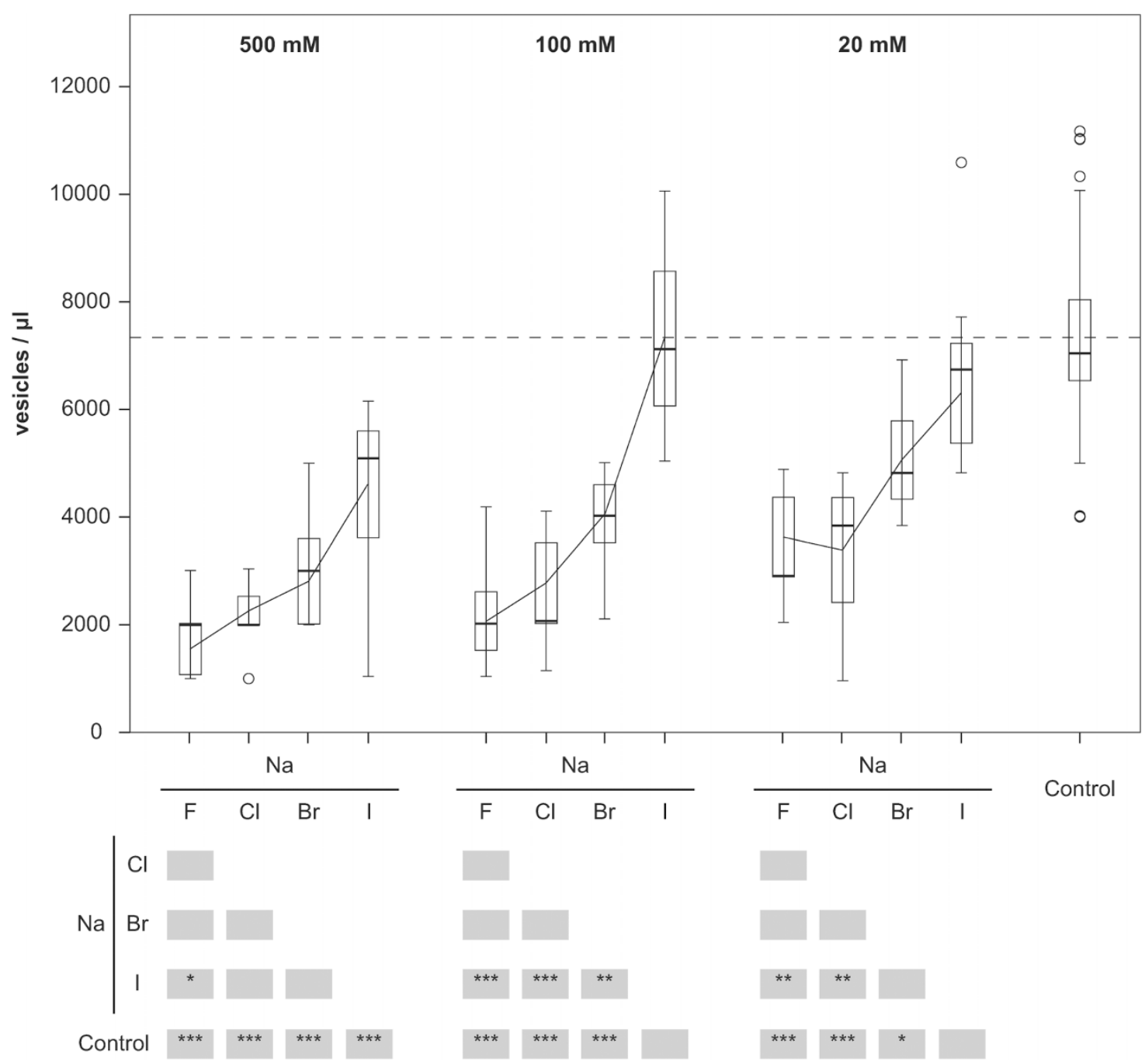

Figure $2 \mid$ Trends in vesicle yield in presence of 500,100 , and $20 \mathrm{mM}$ of $\mathrm{NaF}, \mathrm{NaCl}, \mathrm{NaBr}$, and $\mathrm{NaI}$. The vesicle concentration correlates positively with the Hofmeister series, i.e. down the halogen group. A one-way analysis of variance with Tukey's honestly significant difference post hoc test was used for all samples. Significant differences of the pairwise tests are listed in tabular form below the box plot with levels of significance of $p<0.05(*), p<0.01(* *)$, and $p<0.001(* * *)$. Sample size for each sodium halide: $\mathrm{n}=7$; sample size for the positive control: $\mathrm{n}=48$. The dashed line represents the mean value of positive control group, i.e. in the absence of electrolytes. Solid line segments connect the mean values of neighbouring sodium halides.

electrolytes, vesicles were found to be stable for hours. Due to technical limitations, the surfaces of the Neubauer cell counting chamber were untreated and as a consequence the manual vesicle counting methodology had to be adjusted accordingly. Because events of lysis were not observable during automated vesicle counting, it is reasonable to consider the manual vesicle count for the samples of the positive control, as the measurement the most reliable. Statistical analysis did not reveal any significant differences between automatic and manual vesicle counting. However, a closer look at the results reveals for most of the electrolytes a slightly reduced number of manual counts when compared to automatic vesicle counting in the presence of salt and the opposite effect in the absence of salt, i.e. for the positive control (cf. Fig. 1). From the comparison of automatic and manual counting for the positive control one may assume that there is vesicle lysis during the automatic counting, and the actual vesicle yield may therefore be underestimated in this study. However, the opposite trend in the presence of salt indicates that the shorter measuring time of the automatic counting (1 min) compared to the manual counting $(4 \pm 1 \mathrm{~min})$ compensates for this shortcoming.

Collins and Washabaugh ${ }^{4}$ reported that anions by far dominate cations in terms of Hofmeister effects. The results reported here are in perfect accordance to these findings because the span of vesicle concentration is larger along the halides (cf. Figs. 2 and 3 ) than along the alkali metals (cf. Fig. 4). In addition, only iodide - independent of the alkali metal cation, i.e. sodium or potassium - does not negatively affect vesicle preparation if compared to the positive control at concentrations of 100 and $20 \mathrm{mM}$. This is consistent with results we reported previously in the context of DNA-mediated self-assembly of vesicles ${ }^{58}$.

Our results reveal a general trend in the effect of halogens and alkali metals on vesicle preparation. The most hydrophobic (i.e. the most chaotropic) anion affects least the vesicle preparation. Interestingly, for cations the inverse series is obtained in our measurements, with the most hydrophilic (i.e. the most kosmotropic) cation affecting the vesicle preparation the least. This general trend exactly fits the series obtained for the influence of anions and cations on the dipole potential of PC liposomes ${ }^{41}$, the attraction of anions and cations to solid-supported membranes ${ }^{18}$, and the absorption of cations to phosphatidylserine liposomes ${ }^{20}$ and POPC vesicles ${ }^{19}$.

Because a molecular-level understanding of the Hofmeister series is still lacking, one can only speculate about how the tested electrolytes affect the vesicle yield. The anions and cations tested may interfere to different extents with the vesicle preparation method and 


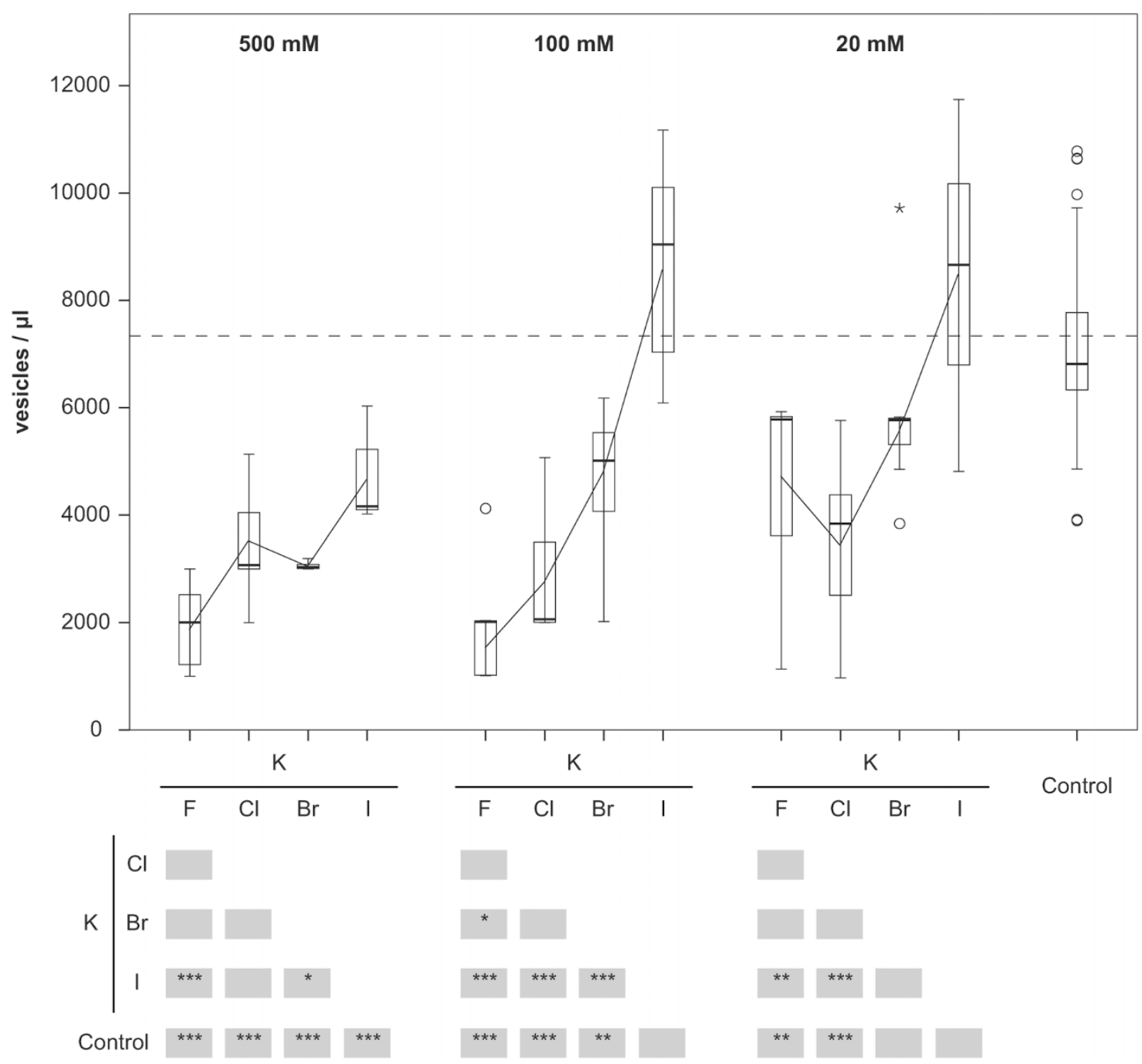

Figure $3 \mid$ Trends in vesicle yield in presence of 500, 100, and $20 \mathrm{mM}$ of $\mathrm{KF}, \mathrm{KCl}, \mathrm{KBr}$, and KI. The vesicle concentration correlates positively with the Hofmeister series, i.e. down the halogen group. A one-way analysis of variance with Tukey's honestly significant difference post hoc test was used for all samples at concentrations of 100 and $20 \mathrm{mM}$. A one-way analysis of variance with Dunnett's T3 post hoc test was used for all samples at a concentration of $500 \mathrm{mM}$. Significant differences of the pairwise tests are listed in tabular form below the box plot with levels of significance of $p<0.05(*), p<0.01(* *)$, and $p<0.001(* * *)$. Sample size for each potassium halide: $\mathrm{n}=7$; sample size for the positive control: $\mathrm{n}=48$. The dashed line represents the mean value of positive control group, i.e. in the absence of electrolytes. Solid line segments connect the mean values of neighbouring potassium halides.

cause reduced vesicle counts when compared to the positive control where no electrolytes are present. Because no changes in the number of vesicles were observed for vesicles stored in microplates for several hours, the stability of vesicles is not negatively affected by the presence of electrolytes once they have been prepared. We introduced a density difference between the equiosmolar solutions of the vesicle lumen and the solution hosting the vesicles. Thus, the vesicles form pellets at the bottom of the microplate wells. This not only allows a manipulation (e.g. exchange of the supernatant, transfer to another vessel) of vesicles in a straightforward manner but also a removal of vesicles, for which an exchange of the solutions inside and outside the vesicle took place (e.g. by a temporal reduction of the membrane integrity) and which as a consequence do not sink to the bottom of the aqueous phase. As Pautot et al. ${ }^{53}$ already reported, vesicles that are equally dense as the surrounding medium remain at the interface between the emulsion phase and the aqueous phase and do not accumulate at the bottom of the aqueous phase. Furthermore, because no differences in the number of water-in-oil emulsion droplets were found for all conditions tested when counted manually (data not shown), we assume that the surface coverage of oil-water interfaces is complete and not affected by the presence of electrolytes, i.e. the water-in-oil droplets in the emulsion phase and the interface between the emulsion phase and the aqueous phase are stabilized by a complete single layer of phospholipids although ions were shown to interact with phospholipid membranes (see Methods for details) ${ }^{10-20}$. Pautot et al. $^{52}$ already discussed the role of the kinetics of interfacial lipid adsorption for the vesicle preparation procedure used in this study. Since the emulsion droplets pass through the interface, they pick up part of the monolayer for completion of a bilayer. One may speculate that different electrolytes affect the absorption of new lipids to replenish the interface differently. Thus, during the relatively fast sedimentation stage, the probability of breakage of emulsion droplets when turning into vesicles, i.e. when emulsion droplets pass the interface between the emulsion phase and the aqueous phase, may depend on the electrolytes present in the aqueous phase.

The combination of the highly parallel vesicle preparation protocol and automatic haemocytometry measurements allowed a reliable quantitative analytical evaluation of the effect of alkali metal halides on the preparation of artificial vesicles prepared by the W/O emulsion transfer method. The sample size of 279 individual measurements allowed solid statistical evaluations. The method reported therefore outnumbers current in vitro and in silico techniques 


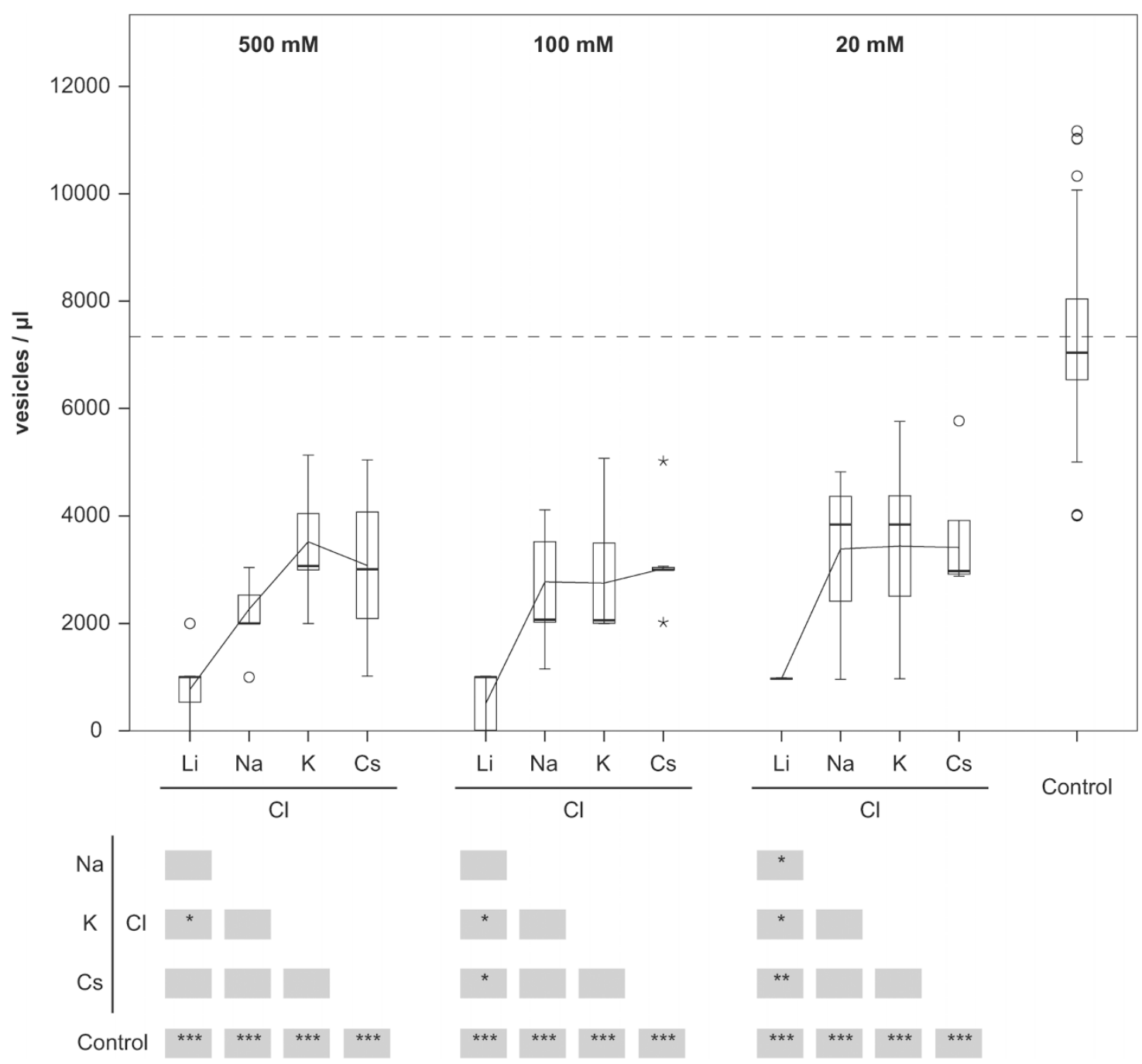

Figure $4 \mid$ Trends in vesicle yield in presence of 500,100 , and $20 \mathrm{mM}$ of $\mathrm{LiCl}, \mathrm{NaCl}, \mathrm{KCl}$, and CsCl. The vesicle concentration correlates positively with the reverse Hofmeister series, i.e. down the alkali metal group, as indicated by the significant differences between $\mathrm{LiCl}$ and $\mathrm{KCl}$ at a concentration of $500 \mathrm{mM}$ and between $\mathrm{LiCl}$ and $\mathrm{CsCl}$ at a concentration of 100 and $20 \mathrm{mM}$. A one-way analysis of variance with Tukey's honestly significant difference post hoc test was used for all samples. Significant differences of the pairwise tests are listed in tabular form below the box plot with levels of significance of $p<0.05$ ( $*$ ), $\left.p<0.01{ }^{* *}\right)$, and $p<0.001(* * *)$. Sample size for each alkali metal chloride: $\mathrm{n}=7$; sample size for the positive control: $\mathrm{n}=48$. The dashed line represents the mean value of positive control group, i.e. in the absence of electrolytes. Solid line segments connect the mean values of neighbouring alkali metal chlorides.

by providing quantitative analytical method of large sample sizes. Our findings reported here are consistent with previous results, i.e. the fitting of automatic and manual vesicle counting, the dominance of anions over cations, and the finding of ion-specific effects as a function of ion size, valency and polarizability following the Hofmeister series or the reverse Hofmeister series, which demonstrates the reliability of our method. Thus, the new semi-automatic quantitative analytical method may provide a significant methodological advance in analysis of free-standing model membranes.

\section{Methods}

The phospholipid POPC (Sigma-Aldrich, Buchs, Switzerland, cat \# P3017) was purchased as a powder and dissolved in chloroform (Scharlau, Barcelona, Spain) to a concentration of $10 \mathrm{mg} / \mathrm{mL}$ upon arrival. After chloroform evaporation (under vacuum, $60 \mathrm{~min}$ ), POPC was dissolved in light mineral oil (Sigma-Aldrich, Buchs, Switzerland, cat \# 330779) to a final concentration of $200 \mu \mathrm{M}$. The POPC in mineral oil was stored under normal atmosphere and used within two days after preparation.

For the vesicle preparation we used a modified version of the $\mathrm{W} / \mathrm{O}$ emulsion transfer method presented by Pautot et al. ${ }^{52,53}$. For a schematic illustration of the vesicle preparation technique and the technical terms (italicized hereafter) used see ${ }^{53}$. For a detailed description of the modifications of the original W/O emulsion transfer method see ${ }^{58}$. Briefly, the modification involved the use of microplates (U96 MicroWell plates, polystyrene clear, U-bottom, Thermo Fisher Scientific,
Langenselbold, Germany) and the density difference between the equiosmolar monosaccharidic intervesicular and the disaccharidic intravesicular aqueous solutions, to detach the vesicles from the interface between intermediate and aqueous phase. The centrifugation to prepare vesicles was performed at $4^{\circ} \mathrm{C}$ and $1500 \times g$ for $10 \mathrm{~min}$. All other steps of the vesicle preparation were performed at room temperature.

All solutions were prepared using high quality water (Milli-Q, Millipore, Brussels, Belgium). Sucrose (99.5\%) and Glucose (99.0\%) were purchase from Sigma-Aldrich (Buchs, Switzerland). The origins and the purity grade of the various alkali metal halides to be investigated were as follows: $\mathrm{LiCl}(99.0 \%), \mathrm{KCl}(99.5 \%), \mathrm{CsCl}(99.0 \%)$, $\mathrm{NaF}(99.0 \%), \mathrm{NaBr}$ (99.5\%), NaI (99.0\%), KF (99.5\%), KBr (99.0\%), and KI (99.0\%) were purchase from Sigma-Aldrich (Buchs, Switzerland); $\mathrm{NaCl}$ (99.5\%) was purchased from Carl Roth (Karlsruhe, Germany).

The emulsion phase (sucrose) as well as the aqueous phase (glucose) was adjusted to $500 \mathrm{mM}$ using a vapour pressure osmometer (Vapro5520, ELITech Group, Puteaux, France). Additionally, to both the emulsion phase and the aqueous phase either 20, 100 , or $500 \mathrm{mM}$ of the alkali metal halides was added and the solutions were readjusted to equiosmolar conditions. Consequently, the emulsion phase as well as the aqueous phase only differed in the density. The W/O emulsion was prepared in microtubes by adding $2 \mu \mathrm{L}$ of the emulsion phase to $200 \mu \mathrm{L}$ of the POPC dissolved in mineral oil. The mixture was mechanically agitated and placed over the intermediate phase (100 $\mu \mathrm{L}$ placed over $100 \mu \mathrm{L}$ of aqueous phase). After incubation (10 min), the centrifugation of the microplates induced vesicle formation. Vesicle preparation was performed in nine replicas for $500 \mathrm{mM}$ and seven replicas for $100 \mathrm{mM}$ and $20 \mathrm{mM}$. The number of replicas for the positive and the negative control was 50 and 4, respectively. 


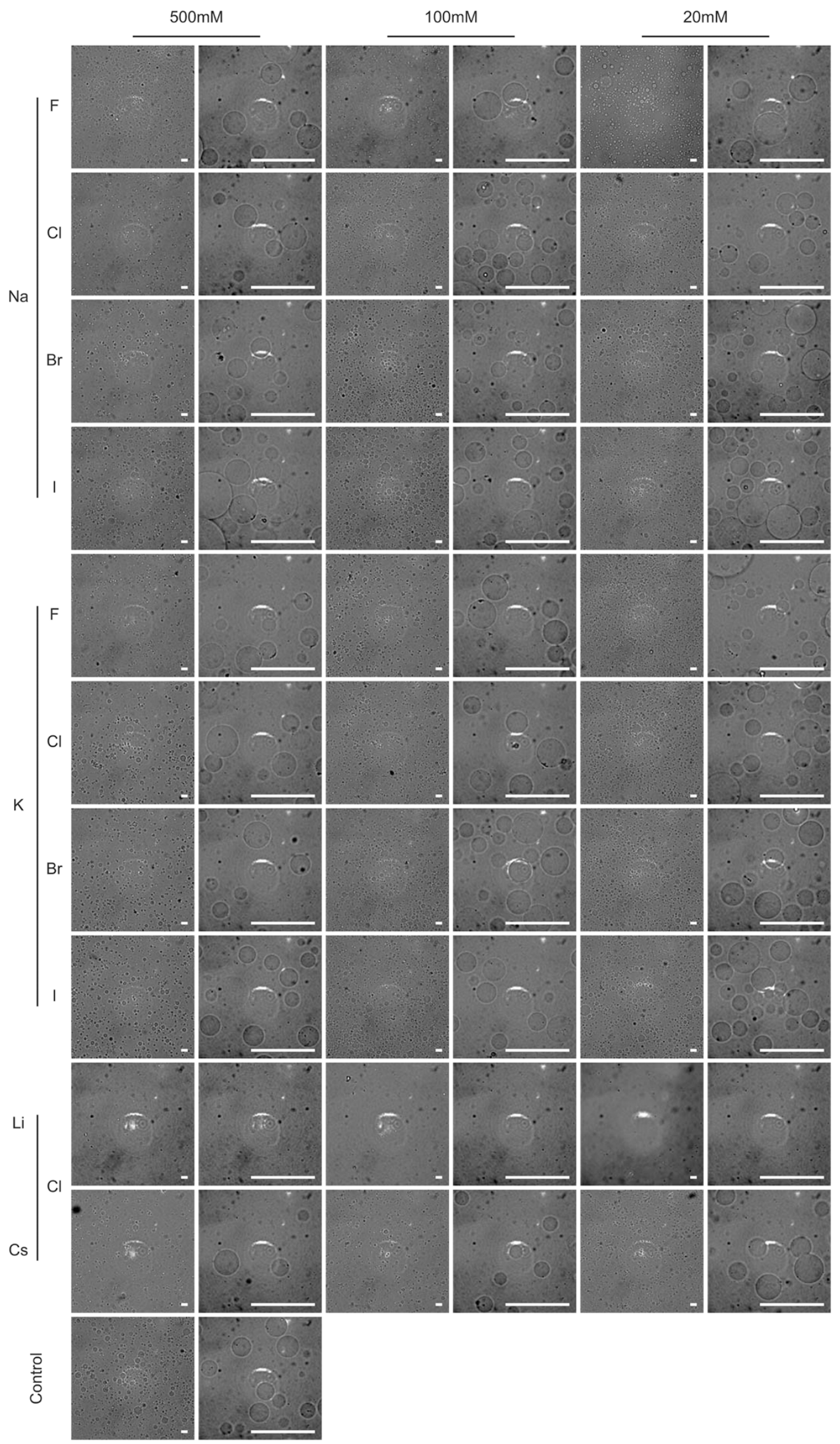

Figure $5 \mid$ Light microscope images of vesicles for all conditions tested. The conditions (i.e. the ions present and their concentration) are listed in the column and row headings. In the positive control experiment, no electrolytes were present. After the transfer from the microplate wells to the microscope slides, within minutes, the vesicles spontaneously sink to the glass surface as a result of the density difference between the vesicle lumen and the hosting medium. Little to no aggregates were found for all conditions. Scale bars: $50 \mu \mathrm{m}$. 
Automatic vesicle counting was performed based on the impedance technology of a fully automated haematology analyser (Sysmex XT-2000iV, Sysmex, Norderstedt, Germany) ${ }^{59}$. To test whether the vesicle preparation method resulted in oil-in-water emulsion droplets or other artefacts that were counted for vesicles incorrectly, in a negative control the same procedure as in the positive control, i.e. electrolytes neither present in the emulsion phase nor in the aqueous phase, was used. The only difference between the negative and positive control was the absence of phospholipids in the mineral oil for the negative control. For all conditions tested seven replicas and 48 replicas for the positive control were analyzed. The manual vesicle counting for the remaining two replicas of each salt of the concentration of $500 \mathrm{mM}$ and of the positive control was performed using an improved Neubauer cell counting chamber mounted to an inverted light microscope at $40 \times$ magnification (Nikon Eclipse TS100). Due to density difference between the equiosmolar monosaccharidic inter- and the disaccharidic intravesicular aqueous solutions, vesicles sank to the bottom of the microplate or the cell counting chamber within seconds. Whereas vesicles were found to be stable for days in polystyrene microplates, contact with the glass surface of the cell counting chamber caused immediate vesicle lysis in the presence of salts. Thus, the manual vesicle counting of the two replicas was limited to a primary square of the cell counting chamber and repeated once for each replica. Automatic and manual vesicle counting was accomplished in one and three to five minutes, respectively. Samples were diluted $1: 6$ for the automatic counting and $1: 60$ for the manual counting with the equiosmolar aqueous phase hosting the vesicles.

For the images of figure 5 , all conditions tested were observed with an inverted light microscope Nikon Eclipse TE2000-S and images captured with a Photometrics Cascade II 512 camera and in-house software. All glass surfaces were treated according to the supplier's recommendations with PlusOne Repel-Silane ES (GE Healthcare, Hillerød, Denmark, cat \# 17-1332-01). The bright field images of figure 5 were automatically contrast-adjusted afterwards (equally across the entire image) using Adobe Photoshop CS, version 8.0.

All statistical analyses were performed according to Janssen and Laatz ${ }^{60}$ by using the PASW statistics software version 18.0.2 (Polar Engineering and Consulting, Nikiski, USA). The comparability of automatic and manual vesicle counting was quantified by an unpaired 2-tailed Welch's $t$ test. A one-way analysis of variance with Tukey's honestly significant difference (HSD) post hoc test, if homogeneity of variance was accepted, or with Dunnett's T3 post hoc test, if homogeneity of variance was rejected, were used to test for differences among the halogens and alkali metals, with levels of significance of $p<0.05, p<0.01$, and $p<0.001$. Normal distribution was tested by a one-sample Kolmogorov-Smirnov test for each salt, each concentration, and each counting method individually. Samples were considered normally distributed if $p>0.05$. Homogeneity of variance of the samples to be compared was tested by using a Levene's test. Inhomogeneity in variance was accepted if $p<0.05$.

The box plots (Figs. 1-4) depict the five-number summaries of the set of observations: sample minimum, lower quartile, median, upper quartile, and sample maximum. Values which are more than one and a half box lengths from either end of the box are flagged as outliers (1.5-3 box lengths from either end of the box: circles; $>3$ box lengths from either end of the box: asterisks)

1. Hofmeister, F. 24. Zur Lehre von der Wirkung der Salze. Naunyn-Schmiedeberg's Archives of Pharmacology 27 (6), 395-413 (1890).

2. Hofmeister, F. Zur Lehre von der Wirkung der Salze. Naunyn-Schmiedeberg's Archives of Pharmacology 24 (4), 247-260 (1888).

3. Zhang, Y. J. \& Cremer, P. S. Chemistry of Hofmeister Anions and Osmolytes. Annu. Rev. Phys. Chem. 61, 63-83 (2010).

4. Collins, K. D. \& Washabaugh, M. W. The Hofmeister Effect and the Behavior of Water at Interfaces. Q. Rev. Biophys. 18, 323-422 (1985).

5. Horowicz, P. Effects of Anions on Excitable Cells. Pharmacol. Rev. 16 (2), 193-221 (1964)

6. Cacace, M. G., Landau, E. M., \& Ramsden, J. J. The Hofmeister series: salt and solvent effects on interfacial phenomena. Q. Rev. Biophys. 30 (3), 241-277 (1997).

7. Baldwin, R. L. How Hofmeister ion interactions affect protein stability. Biophys. J. 71 (4), 2056-2063 (1996).

8. Napper, D. H. Steric stabilization and the Hofmeister series. Journal of Colloid and Interface Science 33 (3), 384-392 (1970).

9. Ninham, B. W. \& Nostro, P. L. Molecular Forces and Self Assembly In Colloid, Nano Sciences and Biology. (Cambridge University Press, 2010).

10. Parsegian, V. A. Ion-Membrane Interactions as Structural Forces. Ann. NY Acad. Sci. 264 (DEC30), 161-174 (1975).

11. Brown, M. F. \& Seelig, J. Ion-Induced Changes in Head Group Conformation of Lecithin Bilayers. Nature 269 (5630), 721-723 (1977).

12. Loosleymillman, M. E., Rand, R. P., \& Parsegian, V. A. Effects of Mono-Valent Ion Binding and Screening on Measured Electrostatic Forces Between Charged Phospholipid-Bilayers. Biophys. J. 40 (3), 221-232 (1982).

13. Tatulian, S. A. Binding of Alkaline-Earth Metal-Cations and some Anions to Phosphatidylcholine Liposomes. Eur. J. Biochem. 170 (1-2), 413-420 (1987)

14. Cunningham, B. A., Gelerinter, E., \& Lis, L. J. Mono-Valent IonPhosphatidylcholine Interactions - an Electron-Paramagnetic Resonance Study. Chem. Phys. Lipids 46 (3), 205-211 (1988).

15. Macdonald, P. M. \& Seelig, J. Anion Binding to Neutral and Positively Charged Lipid-Membranes. Biochemistry 27 (18), 6769-6775 (1988).

16. Roux, M., and M. Bloom Ca-2+, $\mathrm{Mg}-2+, \mathrm{Li}+, \mathrm{Na}+$, and $\mathrm{K}+$ Distributions in the Headgroup Region of Binary Membranes of Phosphatidylcholine and
Phosphatidylserine as Seen by Deuterium NMR. Biochemistry 29, 7077-7089 (1990).

17. Rydall, J. R. \& Macdonald, P. M. Investigation of Anion Binding to Neutral LipidMembranes Using H-2 NMR. Biochemistry 31, 1092-1099 (1992).

18. Garcia-Celma, J. J., Hatahet, L., Kunz, W., \& Fendler, K. Specific anion and cation binding to lipid membranes investigated on a solid supported membrane. Langmuir 23 (20), 10074-10080 (2007).

19. Klasczyk, B., Knecht, V., Lipowsky, R., \& Dimova, R. Interactions of Alkali Metal Chlorides with Phosphatidylcholine Vesicles. Langmuir 26 (24), 18951-18958 (2010).

20. Eisenberg, M., Gresalfi, T., Riccio, T., \& McLaughlin, S. Adsorption of MonoValent Cations to Bilayer Membranes Containing Negative Phospholipids. Biochemistry 18 (23), 5213-5223 (1979).

21. Parsegian, V. A, Rand, R. P., Lipowsky, R., \& Sackmann, E. Chapter 13 Interaction in membrane assemblies in Handbook of Biological Physics (North-Holland, 1995), Vol. Volume 1, Part 2, pp. 643-690.

22. Kunz, W., Henle, J., \& Ninham, B. W. 'Zur Lehre von der Wirkung der Salze' (about the science of the effect of salts): Franz Hofmeister's historical papers. Curr. Opin. Colloid Interface Sci. 9 (1-2), 19-37 (2004).

23. Simon, S. A., Lis, L. J., Kauffman, J. W., \& Macdonald, R. C. Calorimetric and Monolayer Investigation of Influence of Ions on Thermodynamic Properties of Phosphatidylcholine. Biochimica Et Biophysica Acta 375 (3), 317-326 (1975).

24. Hauser, H. et al. Interaction of Ions with Phosphatidylcholine Bilayers. Biochimica Et Biophysica Acta 468 (3), 364-377 (1977).

25. Lis, L. J., Parsegian, V. A., \& Rand, R. P. Binding of Divalent-Cations to Dipalmitoylphosphatidylcholine Bilayers and its Effect on Bilayer Interaction. Biochemistry 20 (7), 1761-1770 (1981).

26. Lis, L. J., Lis, W. T., Parsegian, V. A., \& Rand, R. P. Adsorption of Divalent-Cations to a Variety of Phosphatidylcholine Bilayers. Biochemistry 20 (7), 1771-1777 (1981).

27. Akutsu, H. \& Seelig, J. Interaction of Metal-Ions with Phosphatidylcholine Bilayer-Membranes. Biochemistry 20 (26), 7366-7373 (1981).

28. Altenbach, C. \& Seelig, J. Ca-2+ Binding to Phosphatidylcholine Bilayers as Studied by Deuterium Magnetic-Resonance - Evidence for the Formation of a Ca-2+ Complex with 2 Phospholipidmolecules. Biochemistry 23 (17), 3913-3920 (1984)

29. Oshima, H., Inoko, Y., \& Mitsui, T. Hamaker constant and binding constants of $\mathrm{Ca} 2+$ and $\mathrm{Mg} 2+$ in dipalmitoyl phosphatidylcholine/water system. Journal of Colloid and Interface Science 86 (1), 57-72 (1982).

30. Afzal, S., Tesler, W. J., Blessing, S. K., Collins, J. M., \& Lis, L. J. Hydration force between phosphatidylcholine surfaces in aqueous electrolyte solutions. Journal of Colloid and Interface Science 97 (2), 303-307 (1984).

31. Rappolt, M., Pabst, G., Amenitsch, H., \& Laggner, P. Salt-induced phase separation in the liquid crystalline phase of phosphatidylcholines. Colloid Surf. A-Physicochem. Eng. Asp. 183, 171-181 (2001).

32. Scarpa, M. V., Maximiano, F. A., Chaimovich, H., \& Cuccovia, I. A. Interfacial concentrations of chloride and bromide and selectivity for ion exchange in vesicles prepared with dioctadecyldimethylammonium halides, lipids, and their mixtures. Langmuir 18 (23), 8817-8823 (2002).

33. Jendrasiak, G. L. Halide Interaction with Phospholipids - Proton MagneticResonance Studies. Chem. Phys. Lipids 9 (2), 133-146 (1972).

34. Jendrasiak, G. L., Smith, R., \& Ribeiro, A. A. Chaotropic Anion Phosphatidylcholine Membrane Interactions - an Ultra High-Field NMR-Study. Biochimica Et Biophysica Acta 1145 (1), 25-32 (1993).

35. Loshchilova, E. \& Karvaly, B. Laser Raman Studies of Molecular-Interactions with Phosphatidylcholine Multilayers .2. Effects of Mono-Valent and Divalent Ions on Bilayer Structure. Biochimica Et Biophysica Acta 514 (2), 274-285 (1978).

36. Macdonald, P. M. \& Seelig, J. Anion binding to neutral and positively-charged lipid membranes. Biochemistry 27 (18), 6769-6775 (1988).

37. Epand, R. M. \& Bryszewska, M. Modulation of the Bilayer to Hexagonal PhaseTransition and Solvation of Phosphatidylethanolamines in Aqueous SaltSolutions. Biochemistry 27 (24), 8776-8779 (1988).

38. McLaughlin, S., Bruder, A., Chen, S., \& Moser, C. Chaotropic Anions and SurfacePotential of Bilayer Membranes. Biochimica Et Biophysica Acta 394 (2), 304-313 (1975).

39. Tatulian, S. A. Effect of Lipid Phase-Transition on the Binding of Anions to Dimyristoylphosphatidylcholine Liposomes. Biochimica Et Biophysica Acta 736 (2), 189-195 (1983)

40. Tatulian, S. A., Gordeliy, V. I., Sokolova, A. E., \& Syrykh, A. G. A NeutronDiffraction Study of the Influence of Ions on Phospholipid Membrane Interactions. Biochimica Et Biophysica Acta 1070 (1), 143-151 (1991).

41. Clarke, R. J. \& Lupfert, C. Influence of anions and cations on the dipole potential of phosphatidylcholine vesicles: A basis for the Hofmeister effect. Biophys. J. 76 (5), 2614-2624 (1999)

42. Chapman, D., Peel, W. E., Kingston, B., \& Lilley, T. H. Lipid Phase-Transitions in Model Biomembranes - Effect of Ions on Phosphatidylcholine Bilayers. Biochimica Et Biophysica Acta 464 (2), 260-275 (1977).

43. Cunningham, B. A. \& Lis, L. J. Thiocyanate and Bromide Ions Influence the Bilayer Structural Parameters of Phosphatidylcholine Bilayers. Biochimica Et Biophysica Acta 861 (2), 237-242 (1986).

44. Cunningham, B. A., Lis, L. J., \& Quinn, P. J. The Influence of Mono-Valent Anion on Dipalmitoylphosphatidylcholine Bilayer Phase-Transitions - a Time Resolved X-Ray-Diffraction Study. Molecular Crystals and Liquid Crystals 141 (3-4), 361-367 (1986). 
45. Sanderson, P. W., Lis, L. J., Quinn, P. J., \& Williams, W. P. The Hofmeister Effect in Relation to Membrane Lipid Phase-Stability. Biochimica Et Biophysica Acta 1067 (1), 43-50 (1991).

46. Adriana, P., Bozenna, R. z.-R., \& Marek, L. The effect of selected anions on dipalmitoylphosphatidylcholine phase transitions. Z Naturforsch [C] 57 (7-8), 712-716 (2002).

47. Cunningham, B. A. \& Lis, L. J. Interactive Forces Between Phosphatidylcholine Bilayers in Mono-Valent Salt-Solutions. Journal of Colloid and Interface Science 128 (1), 15-25 (1989).

48. Petrache, H. I., Zemb, T., Belloni, L., \& Parsegian, V. A. Salt screening and specific ion adsorption determine neutral-lipid membrane interactions. Proc. Natl. Acad. Sci. U. S. A. 103 (21), 7982-7987 (2006).

49. Bartucci, R. \& Sportelli, L. Spin-Label EPR Study of the Effects of Monovalent Cations, Anions, and Chaotropics on DPPC Multilayers. Biochim. Biophys. Acta-Biomembr. 1195 (2), 229-236 (1994).

50. Bartucci, R., Belsito, S., \& Sportelli, L. Neutral lipid bilayers interacting with chaotropic anions. Chem. Phys. Lipids 79 (2), 171-180 (1996).

51. Claessens, M., van Oort, B. F., Leermakers, F. A. M., Hoekstra, F. A., \& Stuart, M. A. C. Charged lipid vesicles: Effects of salts on bending rigidity, stability, and size. Biophys. J. 87 (6), 3882-3893 (2004).

52. Pautot, S., Frisken, B. J., \& Weitz, D. A. Production of unilamellar vesicles using an inverted emulsion. Langmuir 19 (7), 2870-2879 (2003).

53. Pautot, S., Frisken, B. J., \& Weitz, D. A. Engineering asymmetric vesicles. Proc. Natl. Acad. Sci. U. S. A. 100 (19), 10718-10721 (2003).

54. Hadorn, M., Burla, B., \& Eggenberger Hotz, P. Towards Tailored Communication Networks in Assemblies of Artificial Cells in LNAI, vol. 5865, edited by Kevin Korb, Marcus Randall, \& Tim Hendtlass (Springer, Heidelberg, 2009) pp. 126-135.

55. Hadorn, M. \& Hotz, P. E. Encapsulated Multi-vesicle Assemblies of Programmable Architecture: Towards Personalized Healthcare in Biomedical Engineering Systems and Technologies, edited by A. Fred, J. Filipe, \& H. Gamboa (Springer-Verlag Berlin, Berlin, 2011), Vol. 127, pp. 141-151.

56. Nishimura, K. et al. Population Analysis of Structural Properties of Giant Liposomes by Flow Cytometry. Langmuir 25 (18), 10439-10443 (2009).

57. Toyota, T. et al. Population study of sizes and components of self-reproducing giant multilamellar vesicles. Langmuir 24 (7), 3037-3044 (2008)

58. Hadorn, M. \& Eggenberger Hotz, P. DNA-Mediated Self-Assembly of Artificial Vesicles. PLoS One 5 (3), e9886 (2010).
59. Weissenbacher, S., Riond, B., Hofmann-Lehmann, R., \& Lutz, H. Evaluation of a novel haematology analyser for use with feline blood. Vet. J. 187 (3), 381-387 (2011).

60. Janssen, J. \& Laatz, W. Statistische Datenanalyse mit SPSS für Windows. (Springer, 2005).

\section{Acknowledgments}

Sample preparation and manual vesicle counting was performed at the Institute of Plant Biology, University of Zurich, Switzerland. Haemocytometry measurements were performed at the Faculty of Veterinary Medicine, University of Zurich, Switzerland. The authors thank Prof. E. Martinoia and Prof. H. Lutz (University of Zurich) for providing laboratory equipment and doctoral candidate B. Burla (University of Zurich) for his technical assistance. The authors thank M. Hanczyc (University of Southern Denmark) for discussion and critical reading of the manuscript. M. Hadorn was partly funded by the Swiss National Foundation Project 200020-118127: 'Embryogenic Evolution: From Simulations to Robotic Applications'. E. Boenzli was supported by a grant obtained from the Promedica Foundation, Chur, Switzerland. The research leading to these results has received funding from the European Community's Seventh Framework Programme (FP7/2007-2013) under grant agreement no. 249032 (Project Acronym: MATCHIT)

\section{Author contributions}

$\mathrm{MH}$ and $\mathrm{PEH}$ conceived and designed the experiments. $\mathrm{MH}$ and $\mathrm{EB}$ performed the experiments. $\mathrm{MH}$ and $\mathrm{PEH}$ analyzed the data. All authors contributed to reagents/ materials/analysis tools. All authors wrote the paper and reviewed the manuscript.

\section{Additional information}

Competing financial interests: The authors declare no competing financial interests.

License: This work is licensed under a Creative Commons

Attribution-NonCommercial-NoDerivative Works 3.0 Unported License. To view a copy of this license, visit http://creativecommons.org/licenses/by-nc-nd/3.0/

How to cite this article: Hadorn, M., Boenzli, E. \& Eggenberger Hotz, P. A Quantitative Analytical Method to Test for Salt Effects on Giant Unilamellar Vesicles. Sci. Rep. 1, 168 DOI:10.1038/srep00168 (2011) 